\title{
MERS-CoV Antibodies in Humans, Africa, 2013-2014
}

\section{Anne Liljander, ${ }^{1}$ Benjamin Meyer, ${ }^{1}$ Joerg Jores, Marcel A. Müller, Erik Lattwein, lan Njeru, Bernard Bett, Christian Drosten, Victor Max Corman}

Dromedaries in Africa and elsewhere carry the Middle East respiratory syndrome coronavirus (MERS-CoV). To search for evidence of autochthonous MERS-CoV infection in humans, we tested archived serum from livestock handlers in Kenya for MERS-CoV antibodies. Serologic evidence of infection was confirmed for 2 persons sampled in 2013 and 2014.

$\mathrm{M}$ iddle East respiratory syndrome coronavirus (MERS-CoV) infection causes severe respiratory illness in humans. Some cases have been sporadic, but others have been part of nosocomial outbreaks mainly on the Arabian Peninsula (1), where dromedary camels widely carry the virus and human infections have been directly linked to contact with camels (2-4). As of January 2016, at least 1,625 acute cases in humans and 586 deaths from MERS$\mathrm{CoV}$ infection have been documented (5). In a geographically comprehensive, age-stratified sample representing the population of Saudi Arabia, antibodies against MERS-CoV were detected in $\approx 0.15 \%$, indicating sporadic infections without severe disease (6).

Antibodies against MERS-CoV have also been detected in dromedaries in several countries in Africa (e.g., Nigeria, Egypt, Kenya) in samples collected as long as 30 years ago (7-10). East Africa harbors $>70 \%$ of the world's dromedary population; predominantly unilateral trade is conducted from Africa to the Arabian Peninsula (11). The basal phylogenetic clustering of viral sequences from camels in Africa suggests an African origin of MERS-CoV $(9,10)$.

To our knowledge, evidence for autochthonous human infections in Africa has not been reported. To search for evidence of previous MERS-CoV infection, we tested

Author affiliations: International Livestock Research Institute, Nairobi, Kenya (A. Liljander, J. Jores, B. Bett); University of Bonn Medical Centre, Bonn, Germany (B. Meyer, M.A. Müller, C. Drosten, V.M. Corman); University of Bern, Vetsuisse Faculty, Bern, Switzerland (J. Jores); EUROIMMUN AG, Lübeck, Germany (E. Lattwein); Ministry of Health, Nairobi, Kenya (I. Njeru); German Centre for Infection Research, Bonn (C. Drosten, V.M. Corman)

DOI: http://dx.doi.org/10.3201/eid2206.160064 archived human serum samples collected from 1,122 livestock handlers in Kenya during 2013 and 2014. This work was done in compliance with national regulations and was approved by the ethical committee of African Medical Research and Foundation, Kenya (AMREF-ESRC P65/2013).

\section{The Study}

The serum samples were collected as part of a household survey conducted during 2013-2014 in 2 eastern counties of Kenya, Garissa and Tana River (online Technical Appendix, http://wwwnc.cdc.gov/EID/article/22/6/16-0064Techapp1.pdf). Of those for whom information about sex and age was known, 603 were female and 407 were male, and median age was 27 (range 5-90) years. Occupational data were available for $650(57.9 \%)$ participants; the 3 largest occupational groups represented were pastoralist (20.6\%), farmer (17.0\%), and student (11.4\%). The households of nearly all participants kept or owned livestock, mainly goats, sheep, cattle, and donkeys. Although camel husbandry was not common among participants, camels are widespread in this region. The average camel density (calculated on the basis of census data from 2000-2013) was 1.68 and 1.98 camels $/ \mathrm{km}^{2}$ in Garissa and Tana River County, respectively (7).

We analyzed serum samples for antibodies against MERS-CoV by using a commercial anti-MERS-CoV recombinant ELISA (rELISA; EUROIMMUN AG, Lübeck, Germany), which is based on the recombinant MERSCoV spike protein subunit 1 and specifically detects IgG. Samples were tested at a dilution of 1:100; an optical density ratio of 0.3 was set as a cutoff $(6,12)$. The assay conditions used were the same as those used during a nationwide serologic study in Saudi Arabia (6). A total of 16 $(1.40 \%)$ samples had positive results by rELISA (Table, Figure 1). The proportion of seropositive specimens in both counties in Kenya did not differ significantly (Fisher exact test, $\mathrm{p}=0.07$ ).

We subsequently tested all samples positive by rELISA by using a highly specific MERS-CoV plaque-reduction neutralization test (PRNT) as recommend by the World Health Organization $(6,13)$. Of note, the MERS-CoV strain EMC/2012 used for PRNT may genotypically differ from putatively circulating MERS-CoV strains from Africa. However, there is no serotypic discrimination between strains because the ability of human serum to neutralize

${ }^{1}$ These authors contributed equally to this article. 
Table. Seropositivity for Middle East respiratory syndrome coronavirus in samples from humans in Kenya, 2013-2014*

\begin{tabular}{|c|c|c|c|}
\hline County & No. samples tested & No. (\%) positive by rELISA & $\begin{array}{l}\text { No. (\%) positive by rELISA and with } \geq 50 \% \\
\text { plaque reduction at } 1: 20 \text { dilution }\end{array}$ \\
\hline Garissa & 559 & $4(0.72)$ & 0 \\
\hline Tana River & 563 & $12(2.13)$ & $2(0.36)$ \\
\hline Total & 1,122 & $16(1.43)$ & $2(0.18)$ \\
\hline
\end{tabular}

diverse MERS-CoV strains, including the EMC/2012 strain, does not differ (14).

For the PRNT, dilutions starting at 1:10 were used and titers resulting in 50\% $\left(\mathrm{PRNT}_{50}\right)$ and $90 \%\left(\mathrm{PRNT}_{90}\right)$ plaque reduction were recorded. The 1:20 dilution was the lowest possible diagnostically significant titer (6). The $\mathrm{PRNT}_{50}$ end point was considered confirmation of positivity by rELISA because this end point was found to be most sensitive and still specific during investigations of antibody responses in reverse transcription PCR-confirmed MERS-CoV-positive samples from patients in South Korea (14). Of the 16 samples positive by rELISA, $2(0.18 \%)$ had reproducible MERS-CoV PRNT ${ }_{50}$ titers of 1:20 and 1:40 (Table; Figure 2); 1 of these samples also had a titer of 1:40 when the more stringent $\mathrm{PRNT}_{90}$ end-point criterion was used (Figure 2). For controls, we conducted PRNT testing of 22 samples negative by rELISA from persons originating from the same region as the 2 samples positive by PRNT. None

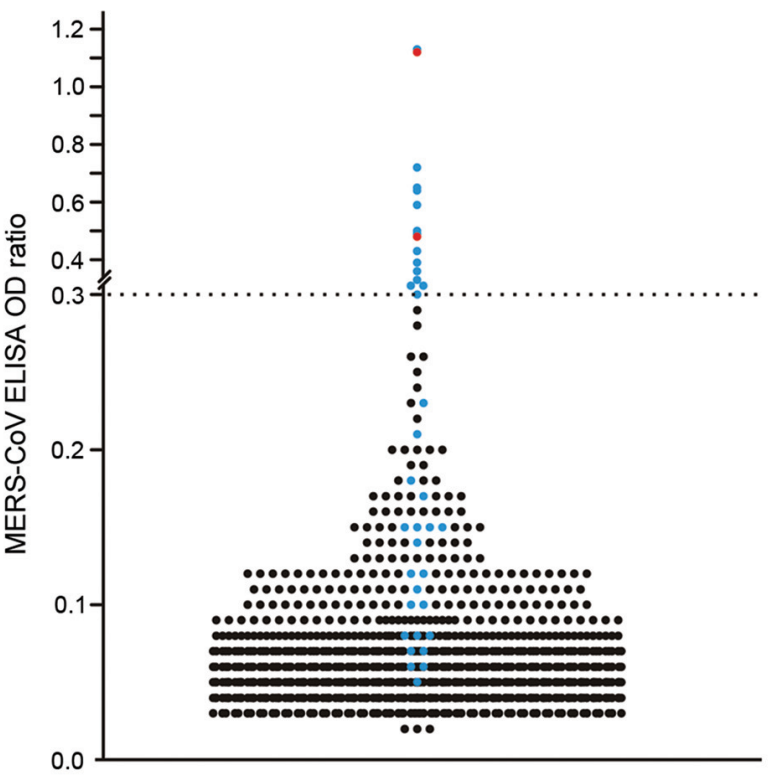

Figure 1. Plot of all individual optical density (OD) ratios obtained from recombinant ELISA testing of human serum samples for Middle East respiratory syndrome coronavirus (MERS-CoV) antibodies, Africa, 2013-2014. All 16 samples exceeding the cutoff of 0.3 and 22 other samples showing an OD ratio below the cutoff were subsequently tested in a plaque-reduction virus neutralization (PRNT) test; these samples are shown in blue, and the 2 samples positive by PRNT are shown in red. The horizontal dashed line represents the cutoff value as determined in a nationwide, cross-sectional serologic study in Saudi Arabia (6). of these 22 samples showed neutralizing activity at a 1:20 dilution (Figure 1).

The 2 samples positive by PRNT were from a woman (26 years of age) and a man (58 years of age) from Tana River County. The woman kept goats, sheep, cattle, and donkeys; the man kept goats and donkeys. Both persons had low antibody titers, and neither reported any recent clinical symptoms, indicating that their MERS-CoV infections probably occurred well before the time of sampling and that the infections may have been mild or subclinical. Because data about persistence of MERS-CoV antibodies after asymptomatic infection are not available, it can only be speculated when and where these infections were acquired. Neither the 2 MERS-CoV antibody-positive persons nor most of the other tested persons owned dromedaries. Nevertheless, camels roam in both counties (7), and humans have regular contact with camels and are likely to consume camel products.

Our study has several limitations. First, we were not able to test samples from persons who had close contact with camels, such as camel pastoralists. Second, although we used well-validated methods and a 2-step approach recommended by the World Health Organization for MERS$\mathrm{CoV}$ diagnostics (13), our results should be confirmed by larger studies that may enable direct virus detection.

\section{Conclusions}

The absence of autochthonous human MERS-CoV infections in Africa has triggered hypotheses regarding differences in disease transmission between Africa and the Arabian Peninsula and has raised doubts regarding the role of camels as a source of infection. Our study provides evidence for unrecorded human MERS-CoV infections in Kenya. The proportion of seropositive specimens that we found is comparable to previously reported proportions of unrecorded infections in the general population in Saudia Arabia $(1.52 \%$ vs. $1.43 \%$ positivity by rELISA and $0.15 \%$ vs. $0.18 \%$ positivity by PRNT for Kenya vs. Saudi Arabia, respectively) (6). Because of an apparently low infection rate and a bias toward reporting severe cases, the discovery of unreported MERS cases requires testing of large sample sizes with well-validated serologic methods (6). Although the number of samples we tested was approximately only one tenth of the number of samples tested during the Saudi Arabia study, the proportion of seropositive specimens may be similar in Kenya and Saudi 


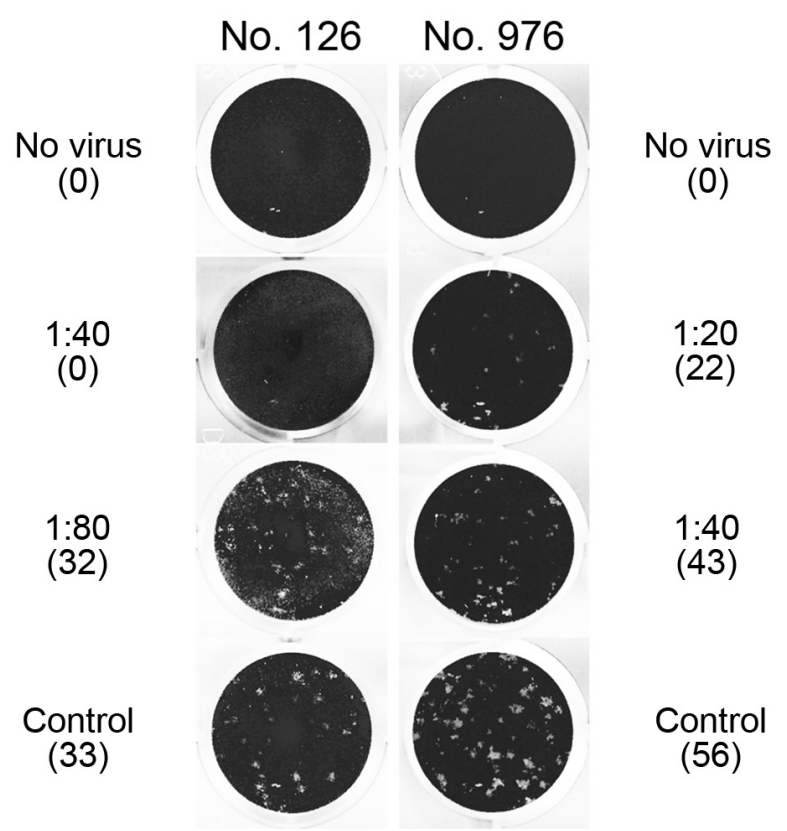

Figure 2. Middle East respiratory syndrome coronavirus (MERS-CoV) plaque-reduction neutralization test (PRNT) results for 2 serum samples positive by recombinant ELISA, showing virus neutralization activity against MERS-CoV strain EMC/2012 exceeding a titer of 1:10. Titers and number of plaques (in parenthesis) are shown next to the corresponding images. Sample no. 976 showed $\geq 50 \%$ plaque reduction up to a titer of $1: 20$, and sample no. 126 showed $\geq 90 \%$ plaque reduction up to a titer of $1: 40$. No serum was added to the control wells. Note that the image cannot represent the morphology and the contrast of plaques that was visible with direct inspection of cell culture plates with an appropriate light source, as was done for these experiments.

Arabia. The lack of a well-developed public health system in parts of Africa could lead to underdiagnosis of clinical cases and would therefore prevent case notification. Moreover, less accessible hospital care might preclude large nosocomial outbreaks as have been observed in countries on the Arabian Peninsula and in South Korea. Other possible explanations for the absence of confirmed and reported clinical cases of MERS-CoV infection in Africa include lesser virulence of strains from Africa and cultural differences that might cause persons of different age ranges to be exposed to the virus.

On the basis of the ability of MERS-CoV to infect a wide range of hosts in cell culture experiments (15), it remains to be excluded that other wild and livestock animals might act as additional sources of human MERS-CoV infection. It is paramount to characterize MERS-CoVs from humans, camels, or tentative other animal hosts in Africa. For increased understanding of any possible differences in pathogenicity and transmission potential, these MERS$\mathrm{CoV}$ strains should be compared with isolates from the Middle East.

\section{Acknowledgments}

We thank all staff members, especially Damaris Mwololo and John Muriuki for their involvement in sampling of human serum; and we thank Tobias Bleicker, Monika Eschbach-Bludau, and Sebastian Brünink for technical assistance.

The work was supported by the German Research Foundation (DFG grant DR772/12-1 to C.D.), the European Commission project PREPARE (contract no. 602525), and the Consultative Group for International Agricultural Research program on Agriculture for Nutrition and Health. The Centrum of International Migration and Development supported the work of A.L. This work was also supported by the project "Dynamic Drivers of Disease in Africa: Ecosystems, Livestock/Wildlife, Health and Wellbeing: REF: NE/J001422/1," funded by the Department for International Development, the Economic and Social Research Council, and the Natural Environment Research Council.

Dr Liljander is a scientist at the International Livestock Research Institute, Kenya. Her current research focus is on the development of new diagnostic tools and vaccines for the progressive control of infectious diseases and the characterization of host-pathogen interactions.

\section{References}

1. Mackay IM, Arden KE. MERS coronavirus: diagnostics, epidemiology and transmission. Virol J. 2015;12:222. http://dx.doi.org/10.1186/s12985-015-0439-5

2. Haagmans BL, Al Dhahiry SH, Reusken CB, Raj VS, Galiano M, Myers R, et al. Middle East respiratory syndrome coronavirus in dromedary camels: an outbreak investigation. Lancet Infect Dis. 2014;14:140-5. http://dx.doi.org/10.1016/S1473-3099(13)70690-X

3. Alraddadi BM, Watson JT, Almarashi A, Abedi GR, Turkistani A, Sadran M, et al. Risk factors for primary Middle East respiratory syndrome coronavirus illness in humans, Saudi Arabia, 2014. Emerg Infect Dis. 2016;22:49-55. http://dx.doi.org/10.3201/ eid2201.151340

4. Gossner C, Danielson N, Gervelmeyer A, Berthe F, Faye B, Kaasik Aaslav K, et al. Human-dromedary camel interactions and the risk of acquiring zoonotic Middle East respiratory syndrome coronavirus Infection. Zoonoses Public Health. 2016.;63:1-9.

5. World Health Organization. Middle East respiratory syndrome coronavirus (MERS-CoV)—Saudi Arabia [cited 2016 Jan 4]. http://www.who.int/csr/don/4-january-2016-mers-saudi-arabia/en/

6. Müller MA, Meyer B, Corman VM, Al-Masri M, Turkestani A, Ritz D, et al. Presence of Middle East respiratory syndrome coronavirus antibodies in Saudi Arabia: a nationwide, cross-sectional, serological study. Lancet Infect Dis. 2015; 15:559-64. http://dx.doi.org/10.1016/S1473-3099(15)70090-3

7. Corman VM, Jores J, Meyer B, Younan M, Liljander A, Said MY, et al. Antibodies against MERS coronavirus in dromedary camels, Kenya, 1992-2013. Emerg Infect Dis. 2014;20:1319-22. http://dx.doi.org/10.3201/eid2008.140596

8. Müller MA, Corman VM, Jores J, Meyer B, Younan M, Liljander A, et al. MERS coronavirus neutralizing antibodies in camels, eastern Africa, 1983-1997. Emerg Infect Dis. 2014;20:2093-5. http://dx.doi.org/10.3201/eid2012.141026

9. Chu DK, Oladipo JO, Perera RA, Kuranga SA, Chan SM, Poon LL, et al. Middle East respiratory syndrome coronavirus 
(MERS-CoV) in dromedary camels in Nigeria, 2015. Euro Surveill. 2015;20:pii=30086. PubMed http://dx.doi.org/ 10.2807/1560-7917.ES.2015.20.49.30086

10. Chu DK, Poon LL, Gomaa MM, Shehata MM, Perera RA, Abu Zeid D, et al. MERS coronaviruses in dromedary camels, Egypt. Emerg Infect Dis. 2014;20:1049-53. http://dx.doi.org/ 10.3201/eid2006.140299

11. Jores J. Middle East respiratory syndrome-coronavirus in camels: an overview for Sub-Saharan and North Africa. Evidence on Demand, UK. 2015. http://dx.doi.org/10.12774/eod_cr.july2015.joresj

12. Drosten C, Meyer B, Muller MA, Corman VM, Al-Masri M, Hossain R, et al. Transmission of MERS-coronavirus in household contacts. N Engl J Med. 2014;371:828-35. http://dx.doi.org/ 10.1056/NEJMoa1405858

13. World Health Organization. Laboratory testing for Middle East respiratory syndrome coronavirus [cited 2015 Jun 30].
http://apps.who.int/iris/bitstream/10665/176982/1/WHO_MERS LAB 15.1 eng.pdf

14. Park SW, Perera RA, Choe PG, Lau EH, Choi SJ, Chun JY, et al Comparison of serological assays in human Middle East respiratory syndrome (MERS)-coronavirus infection. Euro Surveill. 2015;20:pii=30042. http://dx.doi.org/10.2807/1560-7917. ES.2015.20.41.30042

15. Eckerle I, Corman VM, Muller MA, Lenk M, Ulrich RG, Drosten C. Replicative capacity of MERS coronavirus in livestock cell lines. Emerg Infect Dis. 2014;20:276-9. http://dx.doi.org/10.3201/eid2002.131182

Address for correspondence: Christian Drosten, Institute of Virology, University of Bonn Medical Centre, Sigmund-Freud-Str. 25, 53105 Bonn, Germany; email: drosten@virology-bonn.de

\section{April 2015: Emerging Viruses Including:}

- Reappearance of Chikungunya, Formerly Called Dengue, in the Americas

- Hantavirus Pulmonary Syndrome, Southern Chile, 1995-2012

- Animal-Associated Exposure to Rabies Virus among Travelers, 1997-2012

- Evolution of Ebola Virus Disease from Exotic Infection to Global Health Priority, Liberia, Mid-2014

- Population Structure and Antimicrobial Resistance of Invasive Serotype IV Group B Streptococcus, Toronto, Ontario, Canada

- Norovirus Genotype Profiles Associated with Foodborne Transmission, 1999-2012

- Sequence Variability and Geographic Distribution of Lassa Virus, Sierra Leone

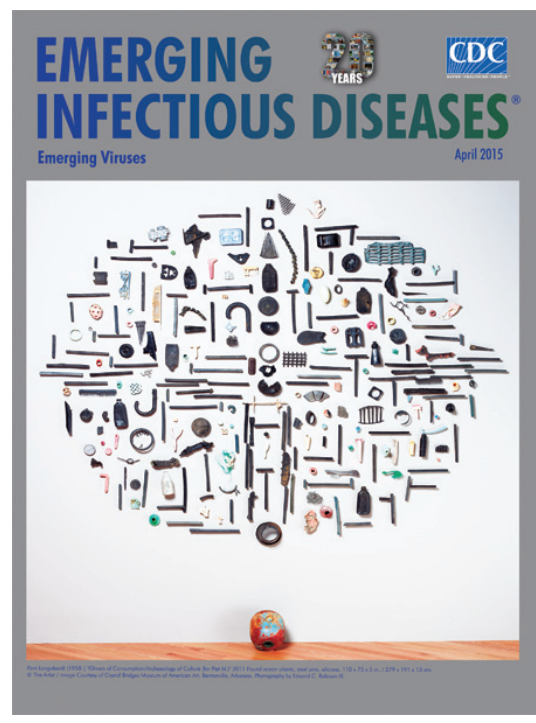

- Deaths Associated with Respiratory Syncytial and Influenza Viruses among Persons $\geq 5$ Years of Age in HIV-Prevalent Area, South Africa, 1998-2009

- Highly Pathogenic Avian Influenza A(H5N1) Virus Infection among Workers at Live Bird Markets, Bangladesh, 2009-2010

- Increased Risk for Group B Streptococcus Sepsis in Young Infants Exposed to HIV, Soweto, South Africa, 2004-2008

- La Crosse Virus in Aedes japonicus japonicus Mosquitoes in the Appalachian Region, United States

- Pathogenicity of 2 Porcine Deltacoronavirus Strains in Gnotobiotic Pigs

- Multidrug-Resistant Salmonella enterica Serotype Typhi, Gulf of Guinea Region, Africa

http://wwwnc.cdc.gov/eid/articles/issue/2 1/4/table-of-contents 